\title{
Micro-oscillations in positive and negative affect during competitive laboratory cycle time trials - a preliminary study
}

\author{
C Rhoden, ${ }^{1} \mathrm{PhD}$; J West, ${ }^{1} \mathrm{MSc} ;$ A Renfree, ${ }^{1} \mathrm{MSc} ;$ M Corbett, ${ }^{1} \mathrm{PhD} ;$ A St Clair Gibson, ${ }^{1,2} \mathrm{PhD}, \mathrm{MD}$ \\ ${ }^{1}$ Institute of Sport and Exercise Sciences, University of Worcester, Henwick Grove, Worcester, United Kingdom \\ ${ }^{2}$ School of Life Sciences, Northumbria University, Northumberland Building, Newcastle Upon Tyne, United Kingdom
}

Corresponding author: C Rhoden (c.rhoden@worc.ac.uk)

\begin{abstract}
Background. By incorporating pre-performance or retrospective recall measurement methods, research has shown positive (PA) and negative affect (NA) to operate as both a precursor to, and as a consequence of performance in line with goal achievement. The extent of this affective change within sport is unclear, as measurement of affect within acute settings has yet to be adopted fully.

Objective. To conduct exploratory research examining affect and goal achievement during self-paced cycling to understand further their role during performance.

Methods. The positive and negative affect schedule (PANAS), Worcester affect scale (WAS) and ratings of goal achievement were completed by seven trained cyclists prior to two separate $20 \mathrm{~km}$ laboratory time trials. The WAS and ratings of goal achievement were also rated during each trial.

Results. Micro-oscillations in affect occurred throughout time trials and to a greater degree where participants were unsuccessful in reaching their goals. Successful trials were characterised by higher PA $(p=0.000)$ and lower NA $(p=0.000)$, with higher goal expectations from the start $(p=0.008)$.

Conclusion. In unsuccessful trials, an overly aggressive start, perhaps due to inaccurate goal setting, led to an inability to maintain performance, with reductions in power output. Further clarification of the catalyst to the performance demise requires a parallel analysis of psychological and physiological parameters. In so doing, a greater understanding of the combined role of affect and goal expectation in pacing and performance will ensue; a benefit to both cyclist and coach alike.
\end{abstract}

S Afr J SM 2014;26(1):20-25. DOI:10.7196/SAJSM.496

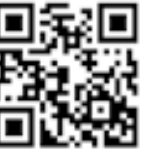

Watson ${ }^{[1]}$ postulated a hierarchical structure of affect with two broad dimensions of positive (PA) and negative affect (NA), reflecting the overall valence of affect in an individual at the higher order level. A lower order level reflects the specific states of mood and emotion experienced by individuals. ${ }^{[2,3]}$ Watson ${ }^{[1]}$ emphasised the notion of a stream of affect, where individuals are continuously experiencing some type of mood. Considering the cyclical nature of moods, he suggested that variables which occur irregularly over time (e.g. exercise) would give rise to unevenly distributed mood fluctuations in comparison to endogenous factors (e.g. the circadian variation in mood). Changes in affect and mood have been observed in both sport and exercise settings, ${ }^{[3,4]}$ revealing the association between affect and performance, success and achievement. In a novel throwing task, NA was significantly higher in failure when compared with successful conditions. ${ }^{[6]} \mathrm{PA}$ predicted performance and was also related to self-efficacy in high school wrestling competitions. ${ }^{[7]}$ More recently, PA was positively correlated to elite climbers' route score.$^{[5]}$ These studies assessed affect at a macro level (pre-performance). We feel that it is also important to consider fluctuations or oscillations in affect at a micro level (within performance), yet this has received limited attention to date and within an exercise setting only. ${ }^{[4]}$
Goal expectations are also pertinent to the understanding of affective responses in sport. In self-regulation theory, monitoring of self- and norm-referenced information enables individuals to make necessary adjustments to minimise the discrepancy between desired and actual behaviour. ${ }^{[8]} \mathrm{A}$ second parallel feedback system, the meta-monitoring function, identifies whether discrepancies are positive, negative or nonexistent, ${ }^{[8]}$ and subsequent affective states ensue. Progress towards a goal at a higher rate than expected (positive discrepancy) leads to PA, and conversely, slower than expected progress (negative discrepancy) leads to increased NA.

Affect changes originating from goal progress interpretation have been shown empirically. Where golfers' actual performance was lower than their goals during a tournament, higher NA and lower PA were experienced ${ }^{[9]}$ though the measurement of affect was undertaken only once and by retrospective recall. Unexpected increases in exercise duration caused runners to become suddenly and significantly more negative, ${ }^{[10]}$ while the negative effects of a psychological crisis (characterised by thoughts about goal disengagement) peaked three-quarters of the way through a marathon and had a negative impact on race performance. ${ }^{[11]}$ Consequently, an individual's perception of likely success while an activity is ongoing becomes an important construct to understand and examine. Moreover, highlighting the role of affective responses and motivation on 
pacing and performance, Baron et al. ${ }^{[12]}$ proposed that athletes would be more likely to maintain or increase exercise intensity when PA was experienced. Where NA was experienced, athletes would have less desire to sustain exercise intensity and a reduction in performance may result. This suggested relationship has obvious implications for time trial performance at the micro level.

Affect operates both as a precursor to, and as a consequence of performance, with actual performance, perceived achievement, success and failure playing additional roles. ${ }^{[5,6]}$ Differing affective experiences may augment or diminish an individual's effort and exercise intensity. Consequently, we sought to examine the relationships between affect, goal expectation and performance by extending previous research protocols to measure affect in parallel with performance measures of time and power output. ${ }^{[5,9]}$ A repeated measures design enabled us to manipulate the analysis of data via the performance outcome, and compare affect profiles between success and failure conditions.

\section{Objectives}

We aimed, via an exploratory research approach: (i) to explore whether micro-oscillations in affect would occur during a competitive time trial environment; and (ii) to assess affect, goal expectations and performance (time and power output) between successful (where cyclists achieved a time goal) and unsuccessful (where cyclists did not achieve a time goal) time trials.

\section{Methods}

\section{Participants}

In an experimental, laboratory-based investigation, seven well-trained cyclists (six males, one female; mean \pm standard deviation (SD) age $32.6 \pm 11.5$ years) performed two $20 \mathrm{~km}$ time trials. Participants were local, competitive club cyclists or triathletes (minimum 2 years' time trial experience). Participants were familiar with $20 \mathrm{~km}$ time trials and regularly paced themselves over this and other distances, enabling us to reproduce a realistic, albeit laboratory, time trial environment. Participants' age, height, weight and $20 \mathrm{~km}$ time trial personal best times did not differ between the successful and unsuccessful groups (Table 1). Prior to testing, participants completed health screening forms and provided full, written, informed consent. All study procedures received institutional ethics committee approval.

\section{Measures}

\section{PANAS}

The positive and negative affect schedule (PANAS) ${ }^{[13]}$ was used to assess PA and NA $30 \mathrm{~min}$ before the time trial. Using a five-point Likert scale from 0 (not at all) to 4 (extremely), participants rated their current responses to ten positive and ten negative adjectives. Internal reliability was demonstrated with a good $\alpha$-coefficient for PA (0.89) and an acceptable $\alpha$-coefficient for NA (0.73).

\section{Worcester affect scale}

The Worcester affect scale (WAS) ${ }^{[14]}$ was used to measure PA and NA at $0.5 \mathrm{~km}$ intervals during the time trial, taking $5-10 \mathrm{~s}$ to complete. Participants rated how they felt 'right now' on two separate ten-point Likert scales: PA ( 1 - not at all positive; 10 - extremely positive) and NA ( 1 - not at all negative; 10 - extremely negative). Preliminary validation indicated the WAS to be related to the PANAS and sensitive in assessing changes in affect during self-paced exercise. ${ }^{[14]}$

\section{Goal expectancy}

Thirty min prior to each trial, participants were asked to identify a time goal based on previous performances and to rate the extent to which they felt they could achieve this goal on a newly created tenpoint Likert scale (1 - not at all; 10 - very much so). Using the same rating scale, participants provided ratings of goal expectancy during the trial at 5, 10 and $15 \mathrm{~km}$ of the total distance. At $20 \mathrm{~km}$, using the same rating scale, participants rated their achievement of goal expectations.

\section{Procedure}

Participants completed two $20 \mathrm{~km}$ time trials, separated by a mean \pm SD of $5.5 \pm 1.9$ days, approaching each time trial as if it were a minor competition to standardise training and nutritional strategies. All participants regularly used the ergometer rig (Kingcycle Ltd, UK) for training purposes and were familiar with the time trial distance in this study; as such, we considered a familiarisation trial unnecessary. At each visit to the laboratory, participants were instructed to ' ... complete the time trial in as fast a time as possible'. Participants recorded their goal for the time trial, provided a goal expectancy rating and then completed the PANAS and WAS. Using their own bicycle mounted and calibrated on the ergometer rig, participants were allowed to view performance feedback: speed, time, power and heart rate. After a self-selected warm-up, comparable with the duration and intensity of their normal pre-time trial routine, participants were given a 5-second countdown. PA and NA were recorded every $0.5 \mathrm{~km}$ using the WAS scales, which were alternated to ensure that no order effects prevailed. Instantaneous power output (W) was recorded visually every $0.5 \mathrm{~km}$ throughout, while ratings of goal expectancy were recorded at 5, 10 and $15 \mathrm{~km}$ during the time trial. Goal achievement and performance time was recorded upon completion of the $20 \mathrm{~km}$. This protocol was the same for both trial 1 and trial 2 .

Table 1. Demographics of the successful and unsuccessful groups

\begin{tabular}{llllll}
\hline Group & $\mathbf{N}$ & $\begin{array}{l}\text { Age (years) } \\
\text { mean } \pm \text { SD }\end{array}$ & $\begin{array}{l}\text { Height (m) } \\
\text { mean } \pm \text { SD }\end{array}$ & $\begin{array}{l}\text { Weight }(\mathbf{k g}) \\
\text { mean } \pm \text { SD }\end{array}$ & $\begin{array}{l}\text { 20 km time trial PB (s) } \\
\text { mean } \pm \text { SD }\end{array}$ \\
\hline Successful & 4 & $29 \pm 12$ & $1.85 \pm 0.07$ & $74.75 \pm 9.18$ & $1963.50 \pm 149.38$ \\
Unsuccessful & 3 & $34 \pm 14$ & $1.79 \pm 0.05$ & $76.83 \pm 2.84$ & $1739.33 \pm 82.25$ \\
$\begin{array}{l}p \text {-value } \\
\text { SD = standard deviation; } \mathrm{PB} \text { = personal best. }\end{array}$ & & 0.642 & 0.286 & 0.725 & 0.069
\end{tabular}



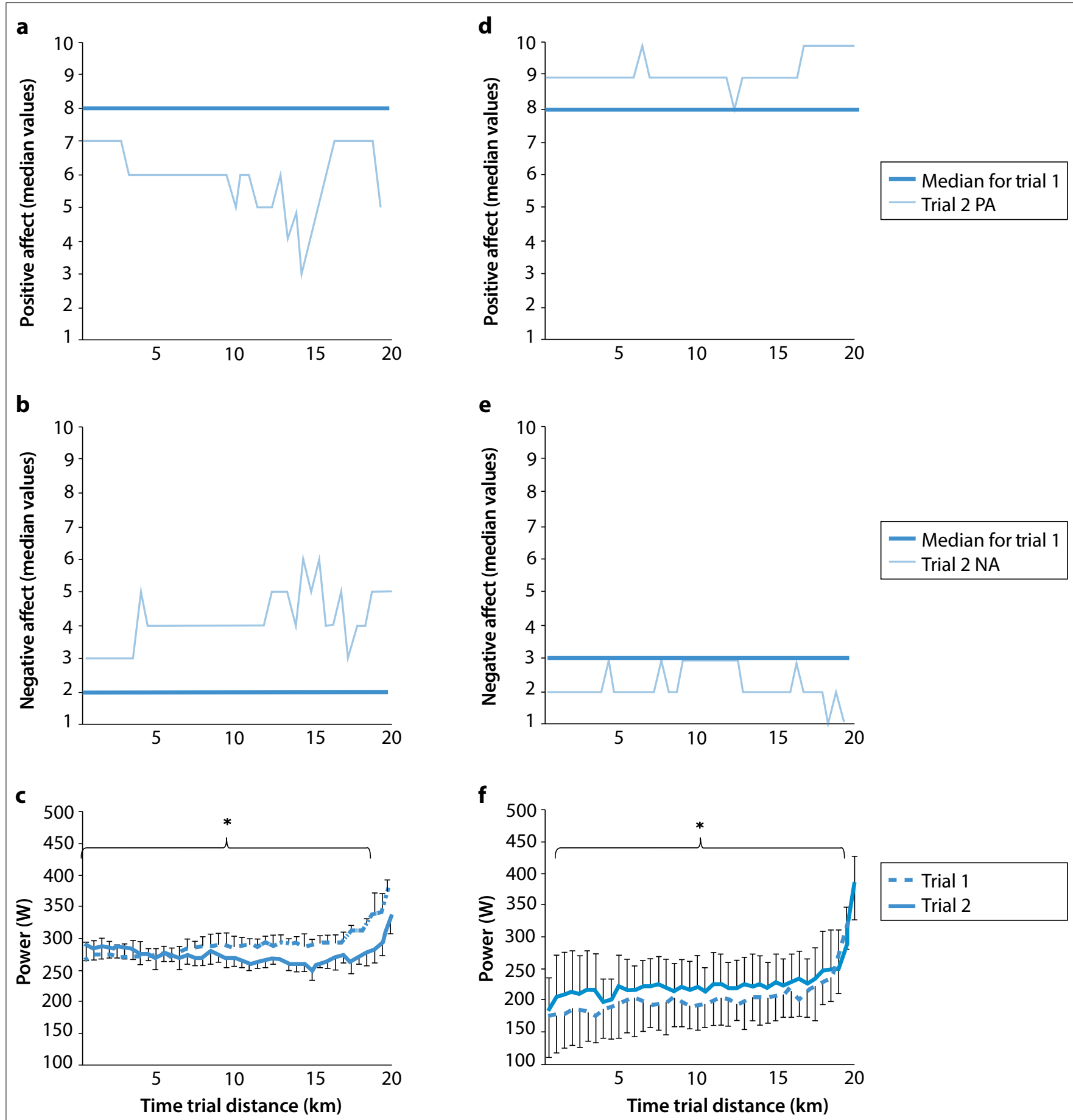

\section{Unsuccessful participants}

\section{Successful participants}

Fig. 1. Worcester affect scale (WAS) median scores and power outputs during the time trial: $(a-c)$ participants who were unsuccessful $(\mathrm{n}=3)$ and $(d-f)$ participants who were successful $(\mathrm{n}=4)$ in trial 2. For $(c)$ and $(f)$, mean \pm standard deviation $(S D)$ power output is displayed; $\left({ }^{*} \mathrm{p}<0.000\right.$; $P A=$ positive affect; $N A=$ negative affect.)

\section{Data analysis}

In this study we undertook psychological examination of a dataset previously published. Renfree et al. ${ }^{[15]}$ analysed the complex interplay between determinants of pacing and performance, and Rhoden and West ${ }^{[14]}$ provided a preliminary validation of the WAS. The current analysis examined affect, goal expectancies and performance between successful and unsuccessful participants in trial 2 only. Prior to the second time trial, all participants reported a time goal, which was to beat their previous time; as such, the expected time goal for trial 2 was anticipated to be less than or equal to the trial 1 performance time. Hence, 


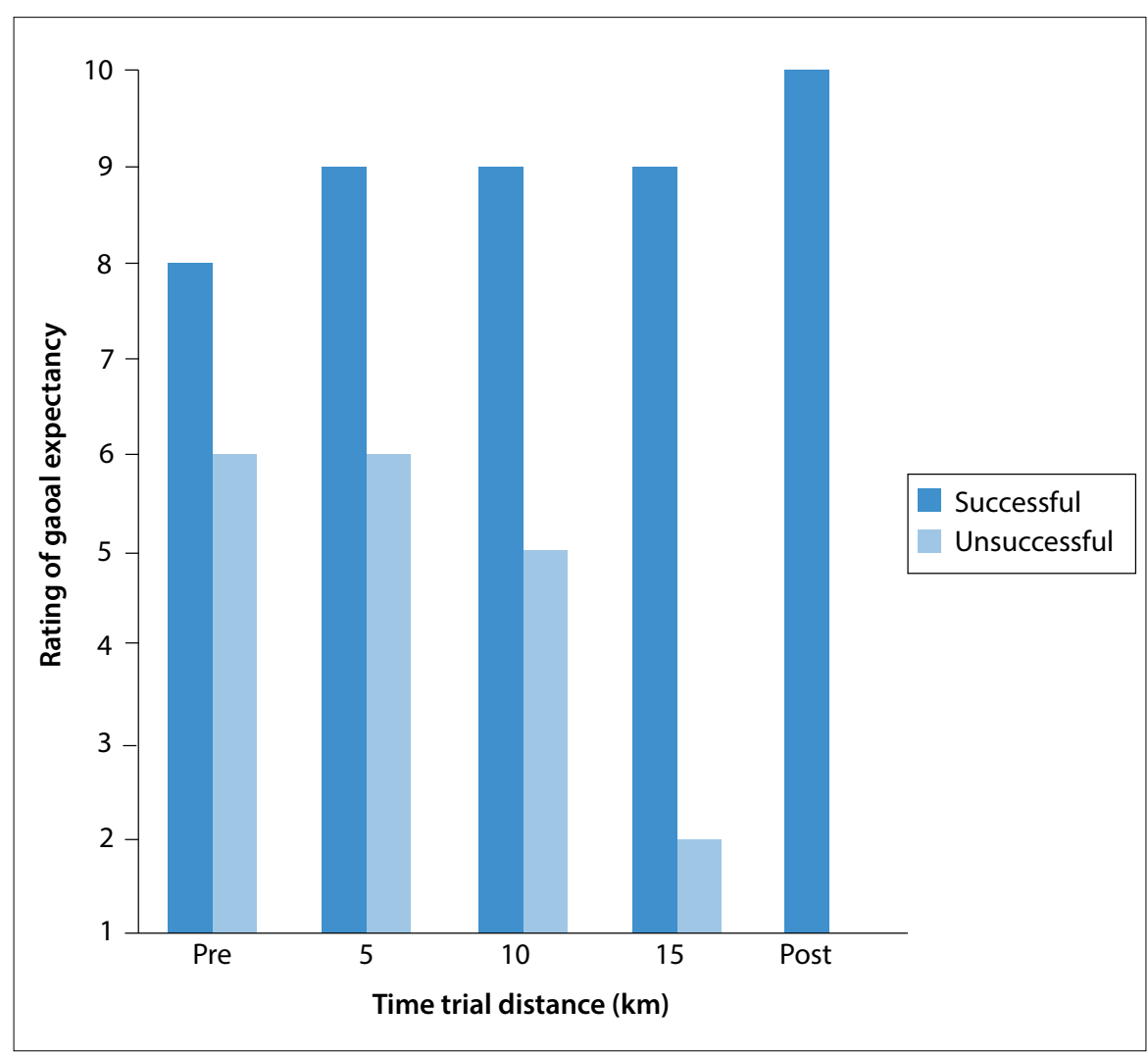

Fig. 2. Ratings of goal expectancy (median scores) between the successful and unsuccessful participants in trial 2 only.

Table 2. Spearman rank order correlations between the WAS and goal expectancy ratings during the time trials

\begin{tabular}{|c|c|c|c|}
\hline \multirow[b]{2}{*}{ Goal expectancy } & \multicolumn{3}{|c|}{ Time trial } \\
\hline & $5 \mathrm{~km}$ & $10 \mathrm{~km}$ & $15 \mathrm{~km}$ \\
\hline \multicolumn{4}{|l|}{$\mathrm{PA}$} \\
\hline Rho & 0.68 & 0.79 & 0.69 \\
\hline$p$-value & 0.007 & 0.001 & 0.006 \\
\hline \multicolumn{4}{|l|}{ NA } \\
\hline Rho & -0.56 & -0.28 & -0.47 \\
\hline$p$-value & 0.038 & 0.316 & 0.094 \\
\hline
\end{tabular}

successful participants were categorised as those who achieved their goal, completing the time trial in the same time as, or faster than their previous time. Unsuccessful participants did not achieve their goal, performing slower than in trial 1. Performance time and power were assessed using paired $t$-tests; however, where distribution curves were non-normal, non-parametric Wilcoxon tests were applied. Mean \pm SD values were used for graphical representation. Affect and goal expectancy respectively. The successful group met their goal expectations, with significantly improved performance times evident in trial 2 (mean \pm SD $1880.00 \pm 160.43 \mathrm{~s}$; improvement $\left.79 \pm 15 \mathrm{~s} ; t=5.08 ; p=0.015 ; \eta^{2}=0.89 ; n=4\right)$. Actual performance times differed from expected times for the unsuccessful participants, who achieved a slower (although not significantly) time in trial $2($ mean \pm SD $1723.33 \pm 10.60 \mathrm{~s}$; deterioration $-38 \pm 15 s ; n=3$ ). PA and NA micro-oscillations occurred throughout the duration of the trials for all participants, and to a greater degree in trial 2 for unsuccessful participants. Unsuccessful participants had lower levels of PA from the outset of the time trial and, in contrast to successful participants, became progressively less positive during the first two-thirds of the time trial $(U=0.0$; $W=820.0 ; Z=-8.0 ; p=0.000 ; r=0.89$; Fig. 1a). They had higher levels of NA from the outset than successful participants $(U=44.0$; $W=864.0 ; Z=-7.55 ; p=0.000 ; r=0.84)$, which peaked at $15 \mathrm{~km}$ (Fig. 1b). Furthermore, unsuccessful participants experienced greater fluctuations in the frequency and magnitude of PA between $10 \mathrm{~km}$ and $17 \mathrm{~km}$ than successful participants (Figs 1a and d). NA also varied considerably for cyclists in the unsuccessful group, with many micro-oscillations again occurring at 12 - 19 km (Fig. 1b). For successful participants, small, periodic fluctuations in NA occurred during the time trial, with a decrease in negativity from $17 \mathrm{~km}$ onwards (Fig. 1e). Pre-trial PANAS scores replicated the pre-trial WAS scores, with unsuccessful participants reporting significantly higher NA (median 7) 30 min prior to the start of the time trial than successful participants (median 3; $U=0.0 ; W=10.0 ; Z=-2.14 ; p=0.032$; $r=0.25$ ), and lower (but not significantly) PA (unsuccessful athletes: median 24; successful athletes: median 32).

Significant differences were observed in the goal expectancies of successful v. unsuccessful cyclists $(U=0.0 ; W=15.0 ; Z=-2.65 ; p=0.008$; $r=0.84$; Fig. 2). Goal expectancy was lower from the outset of the trial for unsuccessful participants, which subsequently decreased compared with the successful participants' ratings. Goal expectancy was consistently positively correlated with PA at 5, 10 and $15 \mathrm{~km}$, and negatively correlated with NA at $5 \mathrm{~km}$ through the time trials (Table 2).

Power output was significantly different between trial 1 and trial 2 for the unsuccessful participants ( $t=5.09 ; p=0.000 ; \eta^{2}=0.39$; Fig. 
1c). They started their slower unsuccessful trial (trial 2) with a higher power output. After $6.5 \mathrm{~km}$ there was a reduction in power output, which remained lower than in their first trial for the remainder of the trial (Fig. 1c). Immediately prior to this point, these participants reported a decrease in PA and an increase in NA (3 - $5 \mathrm{~km}$ ) (Figs la and b). Large oscillations in PA and NA accompanied the continual decrease in power output over the second half of the time trial. In both trials, participants produced an end spurt of similar magnitude $(\sim 70 \mathrm{~W}$ increase) over the last $2.5 \mathrm{~km}$, although actual power was reduced in trial 2 compared with trial 1.

When comparing trial 1 and 2 for successful participants (Fig. 1f), their power output was significantly higher in the successful trial (trial 2) $\left(t=-10.27, p=0.000, \eta^{2=} 0.73\right)$, reflected in a faster performance time. As highlighted previously and in contrast to the unsuccessful participants, PA and NA fluctuated considerably less (Figs $1 \mathrm{~d}$ and e). In both trials 1 and 2, successful participants produced a similar end spurt for the last km.

\section{Discussion}

An important finding of this exploratory research included the microoscillations in PA and NA throughout time trials. The levels of positivity and negativity reported by participants were shown to oscillate - evidence of the dynamic, emotive nature of sport performance. The stream of affect ${ }^{[1]}$ was evident, with PA and NA changing differently between successful and unsuccessful trials, and also varying considerably within each time trial. High levels of PA and lower levels of NA were associated with better performance by the cyclists, further supporting the key role that affect plays in the generation of an optimal sports performance. It is important to note that this pattern emerged throughout the time trials, not only prior to the activity being performed, as has previously been found prior to climbing performance ${ }^{[5]}$ and wrestling. ${ }^{[7]}$

The analysis of affect, goal expectations and performance between the successful and unsuccessful trials yielded another key finding. Goal expectations and affect were closely related throughout the cycle time trial, and in line with previous research, ${ }^{[6]}$ unsuccessful performances were characterised by increased NA, decreased PA and reduced goal expectations from the outset of the trial. Over the course of a time trial, particularly when athletes are striving for a time goal, they make many assessments regarding their performance and rate of goal progress, ${ }^{[16]}$ and paralleling this assessment is the accompanying affect. ${ }^{[9]}$ During unsuccessful trials, cyclists also reported a continual decrease in their beliefs of goal achievement, accompanied by continued high levels of NA, providing further support for the performance-goal discrepancy and resultant affective states. These findings reinforce those of a previous analysis ${ }^{[15]}$ where slow trials (v. fast trials), were characterised by increased NA, decreased PA and decreased goal expectations. Furthermore, slow trials were characterised by increased integrated electromyography yet lower power output, but similar ratings of perceived exertion, which the authors suggested were symptomatic of goal striving. The findings in our analysis demonstrate that the psychological changes in affect are exacerbated by continual failure to achieve goals. Increased variability of affect, both in magnitude and frequency, occurred in participants who experienced continually increasing discrepancies between desired and actual behaviour. We suggest, therefore, that goal striving was occurring and that ongoing assessment of goal achievement mediates the affectperformance relationship.
It is important to note at this point that the cyclists who were unsuccessful started the trial with a higher power output than in their previous trial. Power output subsequently decreased from trial distances of $6.5-15 \mathrm{~km}$. Concurrent with this was increased variability in PA $(10-15 \mathrm{~km})$ and NA (12 - $17 \mathrm{~km})$, similar to the peak of psychological crises reported by marathon runners three-quarters of the way through a race. ${ }^{[11]}$ It is likely that the decreased power output, perception of goal progress and the affective response experienced by the cyclist were crucial at this point in the time trial. A discrepancy between the cyclists' goal expectations and actual performances existed, power output decreased, the cyclists became increasingly more negative and less positive than their more successful counterparts, while large oscillations in affect were evident between $10 \mathrm{~km}$ and $17 \mathrm{~km}$ (Figs la and b), suggesting frequent cognitive appraisals regarding their situation. ${ }^{[17]}$ This combination of factors suggests ineffective goal striving and possible goal disengagement for the unsuccessful cyclists. Undesirable changes in affect and difficulty in goal pursuit can lead to a change in goal-directed behaviour and possible goal disengagement, ${ }^{[18]}$ and where effort is reduced yet the athlete remains committed to their goal, negative feelings of distress result. ${ }^{[16]}$

We were unable to surmise the causal relationships between the psychological and physical performance factors from this study. In exercise settings, increased physical work approaching maximal intensity has been proposed to cause universal negative shifts in affect $^{[4]}$, although the extent to which this holds true for competitive athletes is unclear and it does not fully explain differences between successful and unsuccessful cyclists working maximally in the current study. Baden et al. ${ }^{[10]}$ suggested that fatigue may be an emotional construct, after observing increases in NA concurrent with increases in ratings of perceived exertion. It is plausible that affect changes reported by the unsuccessful cyclists here were the result of increased fatigue $^{[10]}$ from unsustainable power output, and reflected peripheral physiological status. ${ }^{[15]}$ Conversely, the reduced power output may have been the result of increased negativity, with subsequent reduced desire to maintain exercise intensity. ${ }^{[12]}$ Where affective loading (defined by Baron et al. ${ }^{[12]}$ as the difference between NA and PA) surpasses a threshold point (the athlete's highest tolerated affective loading for an expected exercise duration), the desire to sustain high levels of effort decreases, possibly to maintain physiological and emotional homeostasis. The unsuccessful cyclists in this study had higher levels of affective loading (range 3 to -4) than the successful cyclists (range -5 to -9 ); hence this explanation is plausible for this finding. It is also possible that inaccurate assessment of performance expectations, which would have implications for the accuracy of goal setting pre trial, ${ }^{[19]}$ might have accounted for the aggressive start which unsuccessful participants could not maintain for the duration of their second time trial. Both pre-trial goal expectation and affect were suboptimal for the unsuccessful participants, and this perhaps reflects the lack of confidence in the goal set by these participants and their ability to achieve it. Participants were club-level athletes; elite athletes may be better able to assess physiological status prior to time trials and hence be more accurate in the outcome goals that they set.

It is clear that, post trial, the unsuccessful cyclists had low levels of PA and feelings of failure, and this has implications for perceived competence. ${ }^{[7]}$ However, goal disengagement can be an important part of the self-regulation process and produce positive feelings. ${ }^{[16]}$ Indeed, 
Heckhausen et al. ${ }^{[18]}$ stressed that 'the regulatory challenge ... lies in identifying when goal pursuit is maladaptive while it's still ongoing and the individual is fully engaged' (p. 39). Hence future research assessing goal expectancy, goal pursuit and goal striving together with affect and physiological parameters is warranted to identify the threshold point at which goal striving remains beneficial or becomes detrimental to the individual in the longer term.

\section{Study limitations}

Our study was exploratory and we recognise the limitations of the relatively small sample and the variance in participants' training status implied through the differences in performance times between successful and unsuccessful participants. However, the findings show consistent micro-oscillations in affect throughout the time trial, exacerbated by a lack of success, extending previous research (e.g. Gaudreau et al. ${ }^{[9]}$ ) and highlighting an interesting area for future research. Limited research considers the ongoing psychological change during sport, and the self-regulation framework ${ }^{[8]}$ provides a suitable platform to analyse affect, goal expectation and indeed other variables such as self-efficacy. In this study, affect-goal relationships were inextricably linked with power output and performance. Their change has the potential to affect decision-making, and understanding these factors while activity is ongoing is important. ${ }^{[18]}$ As such, we advocate further study of these variables and how they affect each other during performance in larger, controlled studies.

\section{Conclusion}

Micro-oscillations in PA and NA occurred during cycle time trials and were different between successful and unsuccessful trials. Affect profiles more conducive to better performance were characterised by higher levels of PA and lower levels of NA, and these differences occurred from the outset of the trial. Affect and goal expectations were closely related, with goal expectations increasing during successful trials. Successful cyclists reported higher levels of goal expectation from the outset of the trial. Unsuccessful cyclists, although starting trials with higher levels of power, were unable to sustain these levels, which deteriorated up to $15 \mathrm{~km}$, with corresponding decreases in goal expectations and PA and increases in NA. These circumstances may be accounted for by inaccurate goal-setting and assessment of performance expectations prior to the start of the time trial. A full understanding of the catalyst of the time trial performance demise requires further interdisciplinary analysis of both psychological and physiological parameters in parallel. Research examining athletes' experiences of goal striving and goal disengagement would elucidate the factors associated with goal pursuit, which may have implications for the regulation of performance in training and competitive situations from a psychological perspective.

\section{References}

1. Watson D. Mood and Temperament. New York: The Guildford Press, 2000.

2. Watson D, Clark LA. Measurement and mismeasurement of mood: Recurrent and emergent issues. J Pers Assess 1997;68:267-296. [http://dx.doi.org/10.1207/ s15327752jpa6802_4]

3. Watson D, Clark LA. Affects separable and inseparable: On the hierarchical arrangement of the negative affects. J Pers Soc Psychol 1992;62:489-505. [http:// dx.doi.org/10.1037/0022-3514.62.3.489]

4. Ekkekakis P. Pleasure and displeasure from the body: Perspectives from exercise. Cognition Emotion 2003;17:213-239. [http://dx.doi.org/10.1080/02699930244000282]

5. Sanchez X, Boschker MSJ, Llewellyn DJ. Pre-performance psychological states and performance in an elite climbing competition. Scan J Med Sci Sports 2010;20:356-363. [http://dx.doi.org/10.1111/j.1600-0838.2009.00904.x]

6. Walsh J, Crocker PRE, Bouffard M. The effects of perceived competence and goal orientation on affect and task persistence in a physical activity skill. Aust J Sci Med Sport 1992;24:86-90.

7. Treasure DC, Monson J, Lox CL. Relationship between self-efficacy, wrestling performance and affect prior to a competition. Sport Psychol 1996;10:73-83.

8. Carver CS, Scheier MF. On the Self-regulation of Behaviour. New York: Cambridge University Press, 1998.

9. Gaudreau P, Blondin JP, Lapierre AM. Athletes' coping during competition: Relationship of coping strategies with positive affect, negative affect, and performancegoal discrepancy. J Sport Exerc Psychol 2002;3:125-150. [http://dx.doi.org/10.1016/ S1469-0292(01)00015-2]

10. Baden DA, McLean TL, Tucker R, et al. Effect of anticipation during unknown or unexpected exercise duration on rating of perceived exertion, affect and physiological function. Br J Sports Med 2005;39:742-746. [http://dx.doi.org/10.1136/ bjsm.2004.016980]

11. Schüler J, Langens TA. Psychological crisis in a marathon and the buffering effects of self-verbalisations. J Appl Soc Psychol 2007;37:2319-2344. [http://dx.doi.org/10.1111/ j.1559-1816.2007.00260.x]

12. Baron B, Moullan F, Deruelle F, et al. The role of emotions on pacing strategies and performance in middle and long duration sport events. Br J Sports Med 2011;45:511517. [http://dx.doi.org/10.1136/bsjm.2009.059964]

13. Watson D, Clark LA, Tellegen A. Development and validation of brief measures of positive and negative affect: The PANAS scales. J Per Soc Psychol 1988;54:1063-1070. [http://dx.doi.org/10.1037/0022-3514.54.6.1063]

14. Rhoden CL, West J. Affect in sporting activities: A preliminary validation of the Worcester Affect Scale. Sport Science Review 2010;19:71-91.

15. Renfree A, West J, Corbett M, et al. Complex interplay between determinants of pacing and performance during $20 \mathrm{~km}$ cycle time trials. Int J Sports Physiol Perf 2012;7:121-129.

16. Wrosch C, Scheier MF, Carver CS, et al. The importance of goal disengagement in adaptive self-regulation: When giving up is beneficial. Self and Identity 2003;2:1-20. [http://dx.doi.org/10.1080/15298860390129818]

17. St Clair Gibson A, Foster C. The role of self-talk in the awareness of physiological state and physical performance. Sports Med 2007;37:1029-1044. [http://dx.doi.org/01121642/07/0012-1029]

18. Heckhausen J, Wrosch C, Schulz R. A motivational theory of life-span development. Psychol Rev 2010;117:32-60. [http://dx.doi.org/10.1037/a0017668]

19. Micklewright D, Papadopoulou E, Parry D, et al. Perceived exertion influences pacing among ultramarathon runners but post-race mood change is associated with performance expectancy. South African Journal of Sports Medicine 2009;21:167-172. 